\title{
Closure beyond clozapine: successfully averting rebound symptoms in a patient with schizoaffective disorder and agranulocytosis
}

\author{
Alastair Green, Thomas Stephenson, Eromona Whiskey and Sukhi S. Shergill
}

\section{Summary}

'Rebound' or 'withdrawal' symptoms are frequently observed after a sudden discontinuation of clozapine. We describe a patient with treatment-resistant schizoaffective disorder who developed agranulocytosis on clozapine but was successfully switched to treatment with olanzapine with no deterioration in her condition. We put forward three possible theories which may have accounted for the lack of rebound symptoms in this patient: the pharmacological profile of olanzapine, the anticholinergic effects of hyoscine hydrobromide, and the possibility that this patient may not be treatment-resistant and so have a reduced risk of rebound psychosis due to displaying a different pathophysiology.

\section{Declaration of interest}

None.

\section{Keywords}

Clozapine; treatment-resistant schizophrenia; rebound psychosis; agranulocytosis; psychosis.

\section{Copyright and usage}

(c) The Royal College of Psychiatrists 2019. This is an Open Access article, distributed under the terms of the Creative Commons Attribution-NonCommercial-NoDerivatives licence (http://creativecommons.org/licenses/by-nc-nd/4.0/), which permits noncommercial re-use, distribution, and reproduction in any medium, provided the original work is unaltered and is properly cited. The written permission of Cambridge University Press must be obtained for commercial re-use or in order to create a derivative work.
Psychotic illnesses such as schizophrenia and schizoaffective disorder have an annual incidence of 32 cases per 100000 people in England, ${ }^{1}$ with a disproportionate social and economic cost. Antipsychotic medications are first-line pharmacological treatments for such disorders. Clozapine is an atypical antipsychotic with a distinctive pharmacological profile that is uniquely effective in treatment-resistant schizophrenia. ${ }^{2}$ In the UK, National Institute for Health and Care Excellence guidelines recommend that if a patient's illness has not adequately responded to treatment with at least two antipsychotics at appropriate dose and duration, including one second-generation drug, clozapine should then be offered. ${ }^{3}$ Because of its side-effect profile, there are occasions where clozapine must be abruptly stopped. Agranulocytosis is a particular life-threatening concern, affecting around $1 \%$ of patients. ${ }^{4}$ However, given the history of non-response to other antipsychotic medication, there is no established guidance on how to treat patients who are unable to continue taking clozapine. 'Rebound' or 'withdrawal' symptoms are frequently observed after a sudden discontinuation of clozapine; these include prominent psychotic symptoms, anxiety, insomnia, motor restlessness, tardive dyskinesia, altered consciousness, confusion, nausea, diaphoresis and catatonia. ${ }^{6,7}$ Here, we describe a patient who developed agranulocytosis on clozapine but was successfully switched to treatment with olanzapine with no deterioration in her condition. We discuss what this tells us about the possible mechanisms at play.

\section{Case presentation}

A 72-year-old White woman, Ms G, with a 40-year history of treatment-resistant schizoaffective disorder had been managed as an in-patient for the past 6 months under Section 3 of the Mental Health Act. She has described prominent persecutory and grandiose delusional symptoms over the course of her illness, in addition to irritability, mood instability, formal thought disorder, self-neglect and lack of insight. Among Ms G's prominent beliefs are that she is being tricked into paying for her National Health Service care, that she is the victim of identity theft and has several children, and that her psychiatric symptoms are the result of being spiked by drug dealers.

She had been admitted to hospital several times over the course of her illness, demonstrating a relatively consistent pattern of relapse, with prominent episodes of mania with psychotic features. Previously she had been treated with numerous oral and depot antipsychotic medications including trifluoperazine, haloperidol, aripiprazole, risperidone, olanzapine, pipotiazine, paliperidone and zuclopenthixol. A refusal to adhere with oral medications has been a characteristic feature of her illness in the past.

She experienced moderate to severe akathisia and extrapyramidal side-effects, particularly on the left side, on her depot pipotiazine palmitate, which was managed with procyclidine. Previously, she has had evidence of subclinical hypothyroidism.

Ms G has, during her adult life, had intermittent periods of heavy cigarette smoking, although had stopped since this admission to hospital. There is no recent history of drug use or harmful use of alcohol. The available records suggest that Ms G is single and has no children. Her mother died by suicide when she was 1 year old. Her father also died when she was a child.

Ms G was admitted to the National Psychosis Service for a trial of clozapine because of poor symptom control when treated with other antipsychotics, and worsening extrapyramidal symptoms on depot antipsychotics. On admission she was on a weekly depot of zuclopenthixol $(500 \mathrm{mg})$. This was discontinued, and clozapine was titrated up to $250 \mathrm{mg}$ once daily. She responded well initially. An improvement in her mood and a reduction in her irritability and delusional beliefs were noted. However, in her eighth week of treatment her neutrophil levels began to fall, reaching $0.38 \times 10^{9} / \mathrm{L}$ (white cell count $2.03 \times 10^{9} / \mathrm{L}$ ), at which point clozapine was stopped in line with protocol, and she was administered filgrastim $(300 \mu \mathrm{g})$, a human granulocyte colony stimulating factor used to stimulate bone marrow to increase production of neutrophils. She remained neutropenic until the fourth day after stopping clozapine, until neutrophils levels rose to $2.97 \times 10^{9} / \mathrm{L}$. During this time, she was clinically well with no signs of infection. Other medications 
taken at the time were hyoscine hydrobromide ( $300 \mu \mathrm{g}$ three times a day as required) and cholecalciferol (800 units every morning).

Olanzapine was then started (10 mg) at night on the seventh day after clozapine was stopped. There was no deterioration in her mental state during this time off antipsychotic medication. The rationale for this choice of medication was that she had previously responded well to olanzapine during a 3-month period of treatment with subsequent relapse, thought to be related to non-adherence, but not confirmed. At review after 2 weeks on the current trial of olanzapine, no delusions were elicited, there was no evidence of formal thought disorder and she reported a better clarity of mind and reduced hypersalivation than on clozapine. The dose was later increased to $12.5 \mathrm{mg}$ after some increase in delusional beliefs were noted, but she has now been stable for 3 months and is being discharged to a rehabilitation unit.

\section{Discussion}

\section{Averting clozapine rebound symptoms: olanzapine, anticholinergics or neurochemical profile?}

The mechanism causing the withdrawal symptoms associated with a sudden discontinuation of clozapine is unclear. It has been postulated that they are due to clozapine-induced 'supersensitivity' of receptors for dopamine, acetylcholine, gamma-aminobutyric acid and serotonin. Thus, these symptoms occur owing to rebound increase in the corresponding neurotransmitter activity. ${ }^{6}$ Clozapine withdrawal has been suggested to result in psychotic relapse more than some other antipsychotics. Clozapine action via looser binding and rapid displacement at the $\mathrm{D}_{2}$ receptor by endogenous dopamine is another theory that has been posited to explain this rapid withdrawal psychosis. ${ }^{8}$ There is no robust data on incidence of clozapine withdrawal symptoms, but one retrospective study of 28 patients found that $46.4 \%$ of patients experienced rapid deterioration in mental state. ${ }^{9}$ It is therefore an important consideration when circumstances dictate a sudden halt to clozapine therapy. We put forward three possible theories that may have accounted for the lack of rebound symptoms in this patient.

Switching to another antipsychotic medication is a possible approach after abrupt clozapine cessation, although often unsuccessful. Treatment is often symptomatic, using measures such as anticholinergics for dyskinesia and dystonia, ${ }^{10}$ and electroconvulsive therapy for catatonia. ${ }^{11}$ In terms of antipsychotic medication, there is insufficient evidence to favour any particular drug. However, olanzapine in above-licensed doses is most commonly used. ${ }^{5}$ Two randomised controlled trials comparing olanzapine with clozapine in treatment-resistant schizophrenia found olanzapine to have similar efficacy, ${ }^{12,13}$ and this may be owing to its similar pharmacological profile to clozapine. ${ }^{14}$ However, the Maudsley Prescribing Guidelines note that clinical experience of substituting clozapine is disappointing. ${ }^{5}$ One study has specifically compared olanzapine with placebo in patients where clozapine has been discontinued. ${ }^{14}$ The placebo was only given for 3-5 days before commencing olanzapine, but more patients treated with placebo $(24.5 \%)$ than patients treated with olanzapine $(7.5 \%)$ experienced clozapine discontinuation symptoms. Given that the patient in question had a longer, 7-day period between stopping clozapine and starting olanzapine, owing to agranulocytosis, it is possible that she would have been at greater risk of developing rebound symptoms than the patients in the placebo arm of this trial. However, the pharmacological profile of olanzapine may have been an important factor in this case once she had started taking it.

A further reason that may have influenced the successful outcome in this patient is the fact she was taking the anticholinergic medication hyoscine hydrobromide for hypersalivation. One theory of clozapine withdrawal phenomena is that they may be partly due to cholinergic overdrive after sudden removal of the strongly anticholinergic effects of clozapine. ${ }^{6}$ This explains symptoms such as nausea, vomiting, diarrhoea, headache, restlessness, agitation and sweating. In addition to somatic symptoms, anticholinergic withdrawal is also associated with an increase in psychotic symptoms. Patients receiving anticholinergic drugs have been found to be significantly less likely to deteriorate than those not receiving them. ${ }^{9}$ Consequently, anticholinergics have been recommended to treat relevant withdrawal symptoms both prophylactically and symptomatically. ${ }^{15}$ The other consideration in this patient is the fact that before clozapine she was on a depot zuclopenthixol. This has a half-life of 17-21 days and takes around 3 months to be eliminated. Hence, when the clozapine was stopped, she could still have had residual low levels of zuclopenthixol in her system.

It is also possible that this patient may not be treatment-resistant and so have a reduced risk of rebound psychosis owing to a different pathophysiology. It has been proposed that different treatment responses may represent different subtypes of schizophrenia. ${ }^{16} \mathrm{~A}$ recent meta-analysis ${ }^{17}$ found tentative evidence supporting the hypothesis that treatment resistance may be a separate disorder. In contrast to the dysregulation of the dopamine system in treatment-responsive schizophrenia, resistance was found to be better characterised by abnormalities in the glutamate system and reduction in grey matter. One potential explanation being that this patient may have responded well to olanzapine in the past, but only stopped complying covertly and relapsed because of non-adherence, meaning she may not have been genuinely treatment-resistant in the first place. Whether patients experience withdrawal symptoms after clozapine cessation may be a marker of a distinct neurochemical profile to their condition. However, she had been trialled with depot antipsychotic medication for a sustained period of time and still remained symptomatic, suggesting that this is not the entire story.

Of course, we cannot know for certain how or if these possibilities may have interplayed to produce a successful outcome in this case. However, they do raise important points in the management of clozapine withdrawal. More evidence is needed on the choice of antipsychotic and use of anticholinergics. Furthermore, distinguishing between non-adherence and treatment resistance in patients may be a predictor of who is likely to experience rebound symptoms. Current trials of digital medicine interventions may be helpful in this regard. ${ }^{18}$

Alastair Green (1D), BA, Medical Student, Medical School, St George's, University of London, UK; Thomas Stephenson, BSC, MBBS, Core Psychiatry Trainee, Child and Adolescent Clinical Academic Group, South London and Maudsley NHS Foundation Trust, UK; Eromona Whiskey, BPharm, MRPharmS, Clinical Pharmacist, National Psychosis Unit, Bethlem Royal Hospital, South London and Maudsley NHS Foundation Trust, UK; Sukhi S. Shergill, MBBS, SFHEA, PhD, FRCPsych, Professor of Psychiatry \& Systems Neuroscience, Institute of Psychiatry, Psychology and Neuroscience, King's College London; and Honorary Consultant Psychiatrist, National Psychosis Unit, Bethlem Royal Hospital, South London and Maudsley NHS Foundation Trust, UK

Correspondence: Alastair Green, St George's, University of London, Cranmer Terrace, London, SW17 ORE, UK. Email: m1400086@sgul.ac.uk

First received 28 Nov 2018, final revision 18 Apr 2019, accepted 24 Apr 2019

\section{Consent statement}

Written informed consent was obtained from the patient for publication of this case report.

\section{Funding}

S.S.S. is supported by a European Research Council Consolidator Award (grant number 311686) and the National Institute for Health Research Mental Health Biomedical Research Centre at 
South London and Maudsley NHS Foundation Trust and King's College London. The funders had no role in study design, data collection, data analysis, data interpretation or writing of the report.

\section{Acknowledgements}

The publication of this paper is supported by a grant from The Royal college of Psychiatrists Academic Freedom Fund established by Kenneth R. Kaufman, MD FRCPsych. For further details about the fund please visit: https://www.cambridge.org/core/journals/bjpsych-open/information/instructions-contributors.

\section{References}

1 Kirkbride JB, Errazuriz A, Croudace TJ, Morgan C, Jackson D, Boydell J, et al. Incidence of schizophrenia and other psychoses in England, 1950-2009: a systematic review and meta-analyses. PLOS ONE 2012; 7(3): e31660.

2 Kane J, Honigfeld G, Singer J, Meltzer H. Clozapine for the treatment-resistant schizophrenic: a double-blind comparison with chlorpromazine. Arch Gen Psychiatry 1988; 45(9): 789-96.

3 National Institute for Health and Care Excellence (NICE). Psychosis and Schizophrenia in Adults: Prevention and Management (CG178). NICE, 2014 (https://www.nice.org.uk/guidance/cg178/chapter/1-Recommendations).

4 Atkin K, Kendall F, Gould D, Freeman H, Lieberman J, O'Sullivan D. Neutropenia and agranulocytosis in patients receiving clozapine in the UK and Ireland. $\mathrm{Br} J$ Psychiatry 1996; 169(4): 483-8.

5 Taylor D, Barnes T, Young A. The Maudsley Prescribing Guidelines in Psychiatry (13th edn). Wiley Blackwell, 2018.

6 Verghese C, DeLeon J, Nair C, Simpson GM. Clozapine withdrawal effects and receptor profiles of typical and atypical neuroleptics. Biol Psychiatry 1996; 39 (2): $135-8$.

7 Bilbily J, McCollum B, de Leon J. Catatonia secondary to sudden clozapine withdrawal: a case with three repeated episodes and a literature review. Case Rep Psychiatry 2017; 2017: 2402731.

8 Seeman P, Tallerico T. Rapid release of antipsychotic drugs from dopamine D2 receptors: an explanation for low receptor occupancy and early clinical relapse upon withdrawal of clozapine or quetiapine. Am J Psychiatry 1999; 156(6): 876-84.
9 Seppälä N, Kovio C, Leinonen E. Effect of anticholinergics in preventing acute deterioration in patients undergoing abrupt clozapine withdrawal. CNS Drugs 2005; 19(12): 1049-55.

10 Ahmed S, Chengappa KN, Naidu VR, Baker RW, Parepally H, Schooler NR. Clozapine withdrawal-emergent dystonias and dyskinesias: a case series. $J$ Clin Psychiatry 1998; 59(9): 472-7.

11 Lander M, Bastiampillai T, Sareen J. Review of withdrawal catatonia: what does this reveal about clozapine? Transl Psychiatry 2018; 8(1): 139.

12 Tollefson GD, Birkett MA, Kiesler GM, Wood AJ, Lilly Resistant Schizophrenia Study Group. Double-blind comparison of olanzapine versus clozapine in schizophrenic patients clinically eligible for treatment with clozapine. Biol Psychiatry 2001; 49(1): 52-63.

13 Bitter I, Dossenbach MR, Brook S, Feldman PD, Metcalfe S, Gagiano CA, et al. Olanzapine versus clozapine in treatment-resistant or treatment-intolerant schizophrenia. Prog Neuropsychopharmacol Biol Psychiatry 2004; 28(1): 173-80.

14 Tollefson GD, Dellva MA, Mattler CA, Kane JM, Wirshing DA, Kinon BJ, et al. Controlled, double-blind investigation of the clozapine discontinuation symptoms with conversion to either olanzapine or placebo. J Clin Psychopharmacol 1999; 19(5): 435-43.

15 Shiovitz TM, Welke TL, Tigel PD, Anand R, Hartman RD, Sramek JJ, et al. Cholinergic rebound and rapid onset psychosis following abrupt clozapine withdrawal. Schizophr Bull 1996; 22(4): 591-5.

16 Farooq S, Agid O, Foussias G, Remington G. Using treatment response to subtype schizophrenia: proposal for a new paradigm in classification. Schizophr Bull 2013; 39(6): 1169-72.

17 Gillespie AL, Samanaite R, Mill J, Egerton A, MacCabe JH. Is treatment-resistant schizophrenia categorically distinct from treatment-responsive schizophrenia? A systematic review. BMC Psychiatry 2017; 17(1): 12.

18 Peters-Strickland T, Pestreich L, Hatch A, Rohatagi S, Baker RA, Docherty JP, et al. Usability of a novel digital medicine system in adults with schizophrenia treated with sensor-embedded tablets of aripiprazole. Neuropsychiatr Dis Treat 2016; 12: 2587-94. 